\title{
Identification of novel MYB transcription factors involved in the isoflavone biosynthetic pathway by using the combination screening system with agroinfiltration and hairy root transformation
}

\author{
Md. Abdur Rauf Sarkar ${ }^{1,2,3}$, Satoshi Watanabe ${ }^{1,2}$, Akihiro Suzuki 1,2, \\ Fumio Hashimoto', Toyoaki Anai ${ }^{1}, 2, *$ \\ ${ }^{1}$ The United Graduate School of Agricultural Sciences, Kagoshima University, 1-21-24 Korimoto, Kagoshima 890-0065, \\ Japan; ${ }^{2}$ Faculty of Agriculture, Saga University, 1 Honjo-machi, Saga 840-8502, Japan; ${ }^{3}$ Department of Genetic Engineering \\ and Biotechnology, Faculty of Biological Science and Technology, Jashore University of Science and Technology, Jashore \\ 7408, Bangladesh \\ *E-mail: anai@cc.saga-u.ac.jp Tel \& Fax: +81-952-28-8725
}

Received September 13, 2019; accepted October 25, 2019 (Edited by T. Koezuka)

\begin{abstract}
Soybean isoflavones are functionally important secondary metabolites that are mainly accumulated in seeds. Their biosynthetic processes are regulated coordinately at the transcriptional level; however, screening systems for key transcription factors (TFs) are limited. Here we developed a combination screening system comprising a simple agroinfiltration assay and a robust hairy root transformation assay. First, we screened for candidate MYB TFs that could activate the promoters of the chalcone synthase (CHS) gene GmCHS8 and the isoflavone synthase (IFS) genes GmIFS1 and GmIFS2 in the isoflavone biosynthetic pathway. In the agroinfiltration assay, we co-transformed a LjUbi (Lotus japonicus polyubiquitin gene) promoter-fused $M Y B$ gene with target promoter-fused GUS ( $\beta$-glucuronidase) gene constructs, and identified three genes (GmMYB102, GmMYB280, and GmMYB502) as candidate regulators of isoflavone biosynthesis. We then evaluated the functional regulatory role of identified three MYB genes in isoflavone biosynthesis using hairy roots transformation assay in soybean for the accumulation of isoflavones. Three candidate MYB genes showed an increased accumulation of total isoflavones in hairy root transgenic lines. Accumulation of total isoflavones in the three MYBoverexpressing lines was approximately 2 -to 4 -folds more than that in the vector control, confirming their possible role to regulate isoflavone biosynthesis. However, the significant accumulation of authentic GmCHS8, GmIFS1, and GmIFS2 transcripts could not be observed except for the GmMYB502-overexpressing line. Therefore, the analysis of isoflavone accumulation in transgenic hairy root was effective for evaluation of transactivation activity of MYB TFs for isoflavone biosynthetic genes. Our results demonstrate a simple and robust system that can potentially identify the function of orphan TFs in diverse plant metabolic pathways.
\end{abstract}

Key words: agroinfiltration, co-transformation, hairy root, isoflavone, soybean, transcription factor.

\section{Introduction}

Isoflavones are polyphenolic compounds synthesized by leguminous plants and are distinctly abundant in soybean seeds. Soybean seeds are known to be an excellent source of isoflavones in the human diet. Soy isoflavones are considered to have many human health benefits, e.g. the risk reduction of hormonedependent cancers (Dixon 2004; Peterson and Barnes 1991), post-menopausal symptoms (Nestel et al. 1999), cardiovascular disease (Messina 1999), and osteoporosis
(Potter et al. 1998). In plants, isoflavones play an important role in growth, development, and survival. They regulate symbiosis between leguminous plants and rhizobia by inducing the expression of nodulation genes (Ferguson and Mathesius 2003; Kosslak et al. 1987; Novák et al. 2004). Furthermore, isoflavones induce phytoalexin production, which is vital in plant-microbe interactions during pathogen attack (Aoki et al. 2000; Lygin et al. 2013; Yoneyama et al. 2016).

In soybean, isoflavones are coordinately synthesized by multiple biosynthetic enzymes via a legume-specific

Abbreviations: CHI, chalcone isomerase; CHR, chalcone reductase; CHS, chalcone synthase; DAP, days after pollination; GUS, $\beta$-glucuronidase; IFS, isoflavone synthase; LjUbi, Lotus japonicus polyubiquitin gene; qRT-PCR, quantitative reverse transcription-polymerase chain reaction; TF, transcription factor.

This article can be found at http://www.jspcmb.jp/

Published online December 17, 2019 


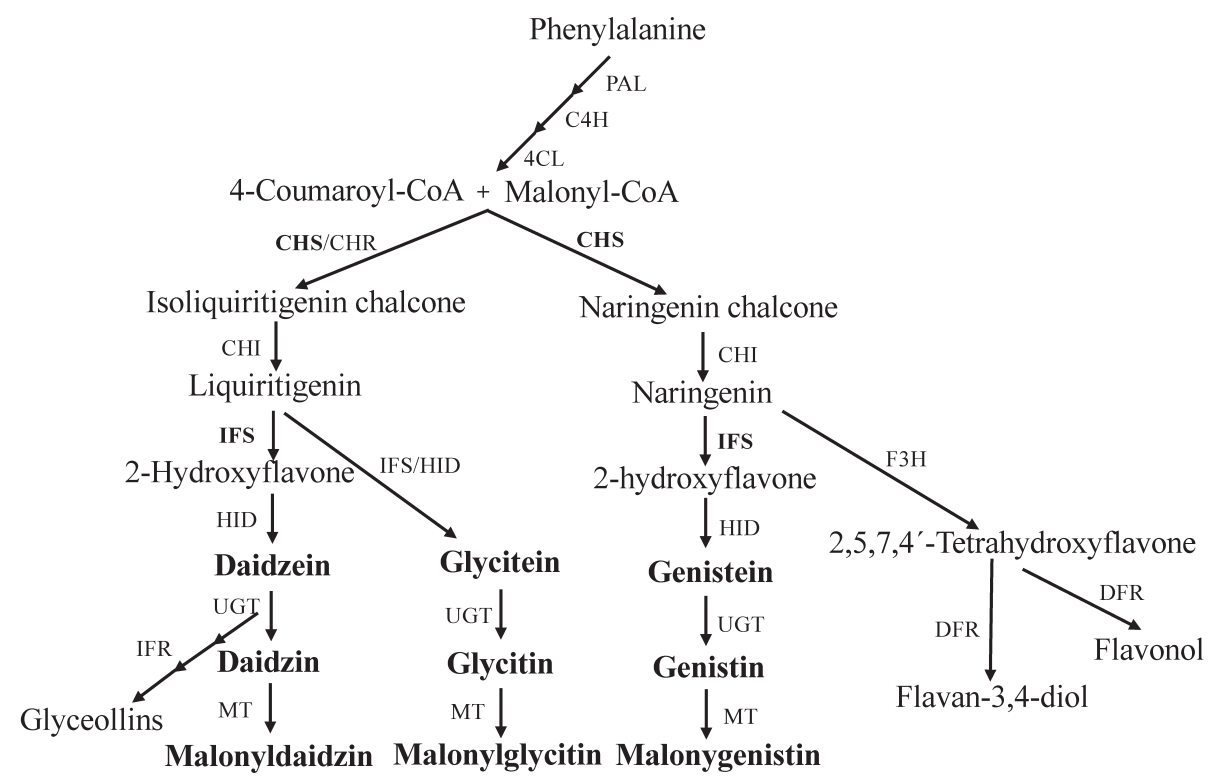

Figure 1. Schematic representation of the isoflavone biosynthetic pathway of soybean. Arrows represent individual enzymatic steps. Abbreviations: 4CL, 4-coumarate-CoA ligase; $\mathrm{C} 4 \mathrm{H}$, cinnamic acid 4-hydroxylase; $\mathrm{CHI}$, chalcone isomerase; CHR, chalcone reductase; CHS, chalcone synthase; DFR, dihydroflavonol 4-reductase; F3H, flavone 3-hydroxylase; HID, 2-hydroxyisoflavone dehydratase; IFR, isoflavone reductase; IFS, isoflavone synthase; MT, isoflavone 7-O-glucoside 6-O-malonyltransferase; PAL, phenylalanine ammonia lyase; and UGT, uridine diphosphate glycosyltransferase. The major isoflavone biosynthetic enzymes and isoflavones are represented as a bold character.

branch of the phenylpropanoid pathway (Figure 1). This branch includes many steps; several biosynthetic genes such as those encoding CHS (chalcone synthase), CHI (chalcone isomerase), IFS (isoflavone synthase), HID (2-hydroxyisoflavone dehydratase), UGT (uridine diphosphate glycosyltransferase), and MT (isoflavone 7-O-glucoside 6-O-malonyltransferase) are involved in the core branch for isoflavone biosynthesis (Akashi et al. 1999; Dastmalchi et al. 2015; Jung et al. 2000; LatundeDada et al. 2001; Steele et al. 1999). The regulatory mechanism of isoflavone biosynthesis through the phenylpropanoid pathway has been extensively studied; the transcriptional levels of isoflavone biosynthetic genes are directly regulated by the ectopic expression of plant transcription factors (TFs) such as MYB, bHLH (basic Helix Loop Helix), bZIP (basic leucine Zipper Protein), WRKY, MADS-box, and WD40 (Grotewold et al. 2000; Nesi et al. 2001; Ramsay and Glover 2005; Yi et al. 2010; Yu et al. 2003).

Through advances in high-throughput sequencing technologies, the annotated genome information from many economically important crops including soybean has become available (Lam et al. 2010; Schmutz et al. 2008). However, functional information on TFs regulating important biosynthetic pathways is still limited because it is difficult to assume the precise function of huge numbers of TFs based on amino acid sequence similarity alone.

In common plant genomes, $\sim 7 \%$ of total transcripts encode TFs. Some of these could be potential tools for manipulation of the phenylpropanoid pathway and improving the levels of isoflavone biosynthesis and accumulation in legume species (Broun 2004). In soybean, 5,671 genes encode putative TFs; MYBs comprise the largest family, representing $14 \%$ of the total TFs (Aoyagi et al. 2014). Manipulating MYB TFs endogenous to legumes could alter the isoflavone biosynthesis by changing the transcriptional activities of the biosynthetic genes.

Using co-expression profiling analysis, one group has recently identified the target genes of orphan TFs (Du et al. 2018; Ye at al. 2017). Others have identified potential TFs related to a specific biosynthesis pathway, lignin biosynthesis in Arabidopsis, by assaying transient expression of target promoters and confirming the results in stable transgenic lines (Newman et al. 2004; Zhou et al. 2009). Nevertheless, it is still not possible to screen TFs involved in a specific biosynthetic pathway in economically important crops in a simple, rapid and reliable way. This is because establishing stable transformants carrying promoter-reporter systems requires regeneration and analysis of numerous lines, which is labor-intensive and time consuming. Therefore, here we developed a method to evaluate the function of orphan TFs by combining two different approaches, a general but preliminary agroinfiltration assay using Nicotiana benthamiana (a close relative of tobacco) (Berger et al. 2007; Kapila et al. 1997; Yang et al. 2000), and a robust hairy root transformation assay in soybean (Kereszt et al. 2007; Ron et al. 2014).

In this study, we succeeded in identifying novel soybean MYB TF candidates that induced the 
transcription of members of the isoflavone biosynthetic pathway, CHS and IFS, in the transient expression assay system. We evaluated the capacity of these candidates, GmMYB102, GmMYB280, and GmMYB502, to activate the isoflavone biosynthetic pathway by using the soybean hairy root transformation system.

\section{Materials and methods}

\section{Plant materials}

Mature seeds and developing seeds at 21, 28, 35, 42, 49, 56, 63, and 70 days after pollination (DAP) were collected randomly from a field-grown soybean [Glycine $\max$ (L.) Merr.] cv. Fukuyutaka at Saga University. The pod wall and seed coat were separated from the developing seed and quickly frozen with liquid nitrogen and stored at $-80^{\circ} \mathrm{C}$ for further analysis.

\section{Construction of plasmids}

The open reading frames of MYB genes were amplified from the cDNA synthesized from developing seeds of c.v. Fukuyutaka by using KOD FX Neo DNA polymerase (TOYOBO, Osaka, Japan). The amplified cDNA fragments were introduced into the entry vector pENTER/D-TOPO (Invitrogen, CA, USA). To prepare the effector constructs, the MYB cDNA fragments were transferred from the pENTER clones to the destination binary vector pUB-GW-GFP by using Gateway technology (Maekawa et al. 2008); the open reading frame fragment of the GUS ( $\beta$-glucoronidase) reporter gene (Jefferson et al. 1986) gene obtained from pCAMBIA1391z was also cloned into pUB-GW-GFP as a positive control. Next, to construct the reporter plasmids, the amplified upstream DNA fragments of CHS8, IFS1, and IFS2 derived from cv. Fukuyutaka were cloned into separate pCAMBIA1391z vectors. We confirmed that all of these constructs (Supplementary Figure S1) had the correct nucleotide sequences. Finally, these constructs were introduced into Agrobacterium tumefaciens strain EHA105 by the freeze-thaw method (Holsters et al. 1978) and their glycerol stocks were stored at $-80^{\circ} \mathrm{C}$ until use. The primer sequences are listed in Supplementary Table S1.

\section{Agroinfiltration assay}

Agroinfiltration was performed as described previously (Yang et al. 2000) with some modifications. Briefly, EHA105 glycerol stock was used to inoculate $4 \mathrm{ml}$ of YEP medium [1\% (v/v) tryptophan, $0.5 \%(\mathrm{v} / \mathrm{v})$ yeast extract, $0.5 \%(\mathrm{w} / \mathrm{v}) \mathrm{NaCl}, \mathrm{pH}$ 7] supplemented with kanamycin $\left(50 \mathrm{mg} \mathrm{ml}^{-1}\right)$. Agrobacteria were grown overnight at $28^{\circ} \mathrm{C}$ with agitation $(200 \mathrm{rpm})$. Then $2 \mathrm{ml}$ of the cultured agrobacteria were used to inoculate $25 \mathrm{ml}$ of the YEP medium supplemented with kanamycin and grown for a further $8 \mathrm{~h}$. Agrobacterial cells were harvested by centrifugation for $10 \mathrm{~min}$ at $2400 \times \mathrm{g}$. The pellet was suspended in $10 \mathrm{mM}$ MES (2-[N-Morpholino] ethanesulfonic acid)$\mathrm{KOH}$ buffer ( $\mathrm{pH}$ 5.6) containing $10 \mathrm{mM} \mathrm{MgCl}_{2}$ and adjusted to a final bacterial concentration of optical density at $600 \mathrm{~nm}$. Finally, freshly prepared $100 \mu \mathrm{M}$ acetosyringone (Sigma-
Aldrich, St. Louis, MO, USA) was added to the agrobacterial suspension, which was kept at room temperature for $1 \mathrm{~h}$ before agroinfiltration to enhance the transient expression efficiency. Nicotiana benthamiana plants were grown in a plant growth chamber under $16 \mathrm{~h}$ light $/ 8 \mathrm{~h}$ dark period at $25^{\circ} \mathrm{C}$ and $60-70 \%$ relative humidity. Four weeks after the plants were placed in the chamber, healthy and green plant leaves were used for the transient assay. The prepared agrobacterial suspension was infiltrated into the underside of the top 3 or 4 leaves by using a blunt-tipped $5 \mathrm{ml}$ plastic syringe (Terumo Corp., Tokyo, Japan) with gentle pressure. The container in which the agroinfiltrated plants were housed was covered with clear plastic wrap until sample collection to avoid severe drying conditions.

\section{Protein extraction and fluorometric GUS assay}

The infiltrated leaves were collected 3 days after infection and homogenized with $650 \mu \mathrm{l}$ of GUS extraction buffer [50 mM $\mathrm{NaH}_{2} \mathrm{PO}_{4}$ (pH 7.0), $0.1 \%$ sarcosyl (N-lauroylsarcosine sodium salt), $10 \mathrm{mM}$ EDTA ( $\mathrm{pH} 8.0$ ), $0.1 \%$ Triton X-100, $10 \mathrm{mM} \beta$-mercaptoethanol, and $20 \%$ methanol]. The total soluble protein content was estimated with the Bradford assay (Bradford 1976) and GUS activity was quantified by a highly sensitive fluorometric assay (Jefferson 1987). Repeated measurements of GUS activities with MUG (4-methylumbelliferyl- $\beta$ - $D$-glucuronide hydrate; SigmaAldrich) as a substrate were taken in triplicate over a kinetic reaction period $(0,15$, and $30 \mathrm{~min})$ at $37^{\circ} \mathrm{C}$. Finally, the reactions were stopped by the addition of $0.2 \mathrm{M} \mathrm{Na}_{2} \mathrm{CO}_{3}$ and fluorescence was measured with the excitation wavelength of $356 \mathrm{~nm}$ and the emission wavelength of $448 \mathrm{~nm}$ using a fluorescence spectrometer (Infinite 200 Pro, Tecan, Männedorf, Switzerland).

\section{Isoflavone extraction and high performance liquid chromatography analysis}

For extraction of isoflavones from developing seeds, each sample was freeze-dried and ground into a fine powder with a Multi-beads Shocker (Yasui Kikai, Osaka, Japan). Precisely $100 \mathrm{mg}$ of fine powder was extracted with $1 \mathrm{ml}$ of $0.1 \%$ acetic acid $(\mathrm{v} / \mathrm{v})$ in $70 \%$ ethanol $(\mathrm{v} / \mathrm{v})$, vortexed, and sonicated for $30 \mathrm{~min}$. The samples were centrifuged at $4^{\circ} \mathrm{C}, 14,000 \times \mathrm{g}$ for $1 \mathrm{~min}$. The extraction procedure was then repeated twice and the supernatant fractions were combined and filtered through a $0.45 \mu \mathrm{m}$ filter. The extracts were analyzed by high performance liquid chromatography (HPLC) on a Hydrosphere C18 column (50 mm length $\times 4.6 \mathrm{~mm}$ internal diameter; YMC Co. Ltd., Kyoto, Japan) at $35^{\circ} \mathrm{C}$. The samples were separated with a linear gradient of solvent A $(0.3 \%$ acetic acid) and solvent B $(0.3 \%$ acetic acid and $0.5 \%$ acetonitrile) at a flow rate of $1.5 \mathrm{ml} \mathrm{min}^{-1}$. The isoflavones were detected by measuring absorbance at $254 \mathrm{~nm}$, and isoflavone peaks were confirmed by comparison with the retention time of nine authentic isoflavone standards (genistin, daidzin, glycitin, genistein, daidzein, glycitein, malonylgenistin, malonyldaidzin, and malonylglycitin; Wako, Osaka, Japan). For extraction of isoflavones from soybean 
transgenic hairy roots, we measured the fresh weight of the roots, froze them in liquid nitrogen, and then ground them into a fine powder by using the Multi-beads Shocker. The powders were used as initial materials for isoflavone extraction. Subsequently, extraction and analysis were performed as above.

\section{Soybean hairy root assay}

Soybean transgenic hairy roots were developed according to the method of Chen et al. (2018) by using soybean cv. Toyoshirome with some modifications. To generate the hairy roots, the effector constructs and the empty vector pUB-GW-GFP as described above were transformed into the Agrobacterium rhizogenes strain LBA1334. Independent positive transgenic hairy root lines were selected by visualization of strong GFP (green fluorescent protein) signals, and were then used for measurement of isoflavones content and gene expression analysis.

\section{Quantitative reverse transcription-polymerase chain reaction analysis}

Total RNA was isolated from developing seeds and transgenic hairy roots of soybean (fresh weight, $50-100 \mathrm{mg}$ ) by using an RNeasy Plant Mini Kit (QIAGEN, Hilden, Germany). The first-strand cDNA was synthesized from $1 \mu \mathrm{g}$ of total RNA with a ReverTra Ace kit (TOYOBO), and oligo(dT)20 primer. cDNA (10 ng) was used as the template for quantitative reverse transcription-polymerase chain reaction (qRT-PCR) analysis with rTaq enzyme and EvaGreen dye (Biotium, Inc., Fremont, CA, USA) and fluorescence intensity was monitored. The qRT-PCR analysis was carried out in 96-well plates using the LightCycler 96 System (Roche Diagnostics K.K., Tokyo, Japan) in a final volume of $10 \mu$ l per well. Each reaction was performed using the following conditions: pre-incubation at $95^{\circ} \mathrm{C}$ for $5 \mathrm{~min}, 40$ cycles at $95^{\circ} \mathrm{C}$ for $15 \mathrm{~s}, 60^{\circ} \mathrm{C}$ for $15 \mathrm{~s}, 72^{\circ} \mathrm{C}$ for $20 \mathrm{~s}$, and melting analysis at $95^{\circ} \mathrm{C}$ for $10 \mathrm{~s}, 65^{\circ} \mathrm{C}$ for $1 \mathrm{~min}$ followed by final extension at $97^{\circ} \mathrm{C}$. Two housekeeping genes, GmACTIN (Glyma.19G147900, for developing seeds) and GmELFa (Glyma.19G052400.1, for hairy roots), were used in each PCR plate for normalization of transcript levels. Data were analyzed with the LightCycler 96 SW 1.1 software package (Roche Diagnostics K.K) and transcript levels were calculated by following the guidelines in Livak and Schmittegen (2001). The gene-specific primer sequences for qRT-PCR are listed in Supplementary Table S2.

\section{Statistical analysis}

We calculated the normalized value (zero mean and variance 1) for each gene expression data and for the total amount of isoflavone molecules (assigned as HPLC data in the figure) during seed development. The package "heatmap.2" was used for the analysis of hierarchical clustering and heat map construction with default parameters. Statistical test for complementation test was conducted with single-factor ANOVA, Tukey post hoc test, $p<0.05$. All analyses were performed in R software (https://www.r-project.org/).

\section{Results}

Transactivation analysis of 12 orphan MYB TFs by agroinfiltration assay

We selected three isoflavone biosynthetic genes, GmCHS8, GmIFS1, and GmIFS2 as major up-regulated target genes in the isoflavone biosynthetic pathway, because the expression of these three genes also showed a higher correlation with isoflavone accumulation in developing soybean seeds (Dhaubhadel et al. 2003, 2007; Yi et al. 2010). We also selected 12 candidate MYB TF genes whose expression highly correlated with GmIFS1 expression by using annotation data and RNA-seq Atlas data in the soybean genome database (https://soybase. org/).

Hereafter, to evaluate the capacity of the 12 orphan R2-R3 type MYB TFs to transactivate the promoters of GmCHS8, GmIFS1, and GmIFS2, we designed a agroinfiltration assay into the leaves of a foreign species, $N$. benthamiana, via agroinfiltration. This assay system consisted of a reporter plasmid containing a GUS gene fused with the target gene (GmCHS8, GmIFS1, or GmIFS2) promoter, and an effector plasmid containing one of the candidate MYB genes or the positive control GmMYB176 gene (Yi et al. 2010) fused with the strong housekeeping L. japonicus polyubiquitin (LjUbq1) promoter (Maekawa et al. 2008).

Out of 13 co-transformations of MYB TFs with GmCHS8 reporter, 7 MYB TFs showed significantly higher GUS activities at $p<0.01$ compared with the reporter alone (Figure 2A and Table 1): i.e., GmMYB4 (2.27-fold [MYB plus reporter/reporter alone]), GmMYB77 (2.28-fold), GmMYB102 (2.76-fold), GmMYB280 (1.82-fold), GmMYB502 (2.26-fold), GmMYB522 (2.09-fold), and GmMYB176 (2.18-fold). In addition, 3 MYB TFs showed significantly higher GUS activities at $p<0.05$ compared with the reporter alone: i.e., GmMYB128 (1.82-fold), and GmMYB250 (1.63fold) (Figure 2A and Table 1). In contrast, GmMYB202 significantly suppressed GUS activity compared with the reporter alone $(0.24$-fold, $p<0.05)$ (Figure $2 \mathrm{~A}$ and Table $1)$.

When GmIFS1 was used as the reporter, cotransformation of seven MYB TFs showed significantly higher GUS activities at $p<0.01$ than transformation with reporter alone (Figure $2 \mathrm{~B}$ and Table 1): i.e., GmMYB4 (1.85-fold), GmMYB77 (1.98-fold), GmMYB102 (1.65-fold), GmMYB250 (1.58-fold), GmMYB280 (1.72-fold), GmMYB502 (1.70-fold), and GmMYB176 (1.77-fold). In contrast, co-transformation with GmMYB202 showed 0.52-fold suppression in GUS activity at $p<0.05$ compared with reporter alone (Figure $2 \mathrm{~B}$ and Table 1).

In co-transformations with GmIFS2 reporter, higher GUS activity was observed for six MYB TFs compared 
A

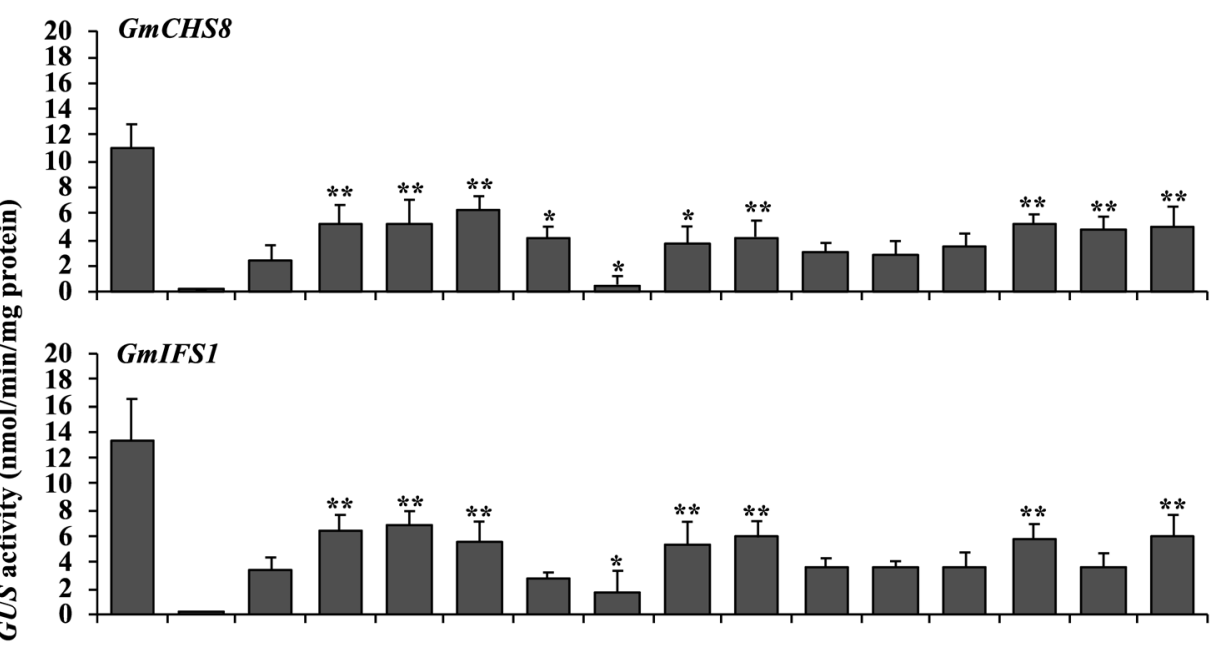

C

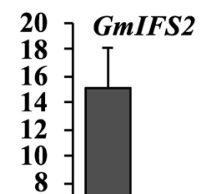

\section{Reporter}

Positive control Negative control

Figure 2. Comparison of the transcriptional activity of soybean MYB TFs in an agroinfiltration assay. The underlined effector construct was used as a positive control MYB TF. The data are presented as means and standard deviations of eight biological replicates. ${ }^{*} p<0.05,{ }^{* *} p<0.01$ versus reporter only (Student's $t$-test).

Table 1. Transactivation of multiple target promoters by transient expression of MYB transcription factors.

\begin{tabular}{|c|c|c|c|c|c|}
\hline Name & MYB type & $\begin{array}{l}\text { Number of amino acid } \\
\text { residues }\end{array}$ & GmCHS 8 & GmIFS1 & GmIFS2 \\
\hline GmMYB4 & R2R3 & 406 & ++ & ++ & + \\
\hline GmMYB77 & R2R3 & 322 & ++ & ++ & ++ \\
\hline GmMYB102 & R2R3 & 275 & ++ & ++ & ++ \\
\hline GmMYB128 & R2R3 & 287 & + & $+1-$ & $+1-$ \\
\hline GmMYB202 & R2R3 & 377 & - & - & - \\
\hline GmMYB250 & $\mathrm{R} 2 \mathrm{R} 3$ & 330 & + & ++ & + \\
\hline GmMYB280 & R2R3 & 252 & + & ++ & $+1-$ \\
\hline GmMYB399 & R2R3 & 452 & $+1-$ & $+1-$ & $+1-$ \\
\hline GmMYB415 & R2R3 & 289 & $+1-$ & $+1-$ & $+1-$ \\
\hline GmMYB467 & R2R3 & 320 & + & $+1-$ & - \\
\hline GmMYB502 & R2R3 & 284 & ++ & ++ & + \\
\hline GmMYB522 & R2R3 & 373 & ++ & $+1-$ & $+1-$ \\
\hline GmMYB176 & $\mathrm{R} 1$ & 285 & ++ & ++ & + \\
\hline
\end{tabular}

MYB types are assigned according to Du et al. (2012), Aoyagi et al. (2014) and soybean genome information from http://phytozome.jgi.doe.gov/.,$++ \geq 2$-fold activation; +, $\geq 1$.5-fold activation;-, $\geq 2$-fold suppression and -, $\geq 1$.5-fold suppression of GUS activity compared to the reporter construct only. $+/-$, no change in GUS activity.

with transformation with reporter alone (Figure 2C). The highest increase in GUS activity was obtained with GmMYB77 (2.08-fold, $p<0.01$ ) (Figure 2C and
Table 1). Five other MYB TFs, GmMYB4 (1.36-fold), GmMYB102 (1.36-fold), GmMYB250 (1.50-fold), GmMYB502 (2.09-fold) and GmMYB176 (1.58-fold) 
also showed significantly increased GUS activity (all $p<0.05$; Figure $2 \mathrm{C}$ and Table 1 ). GUS activities were significantly suppressed $(p<0.05)$ by co-transformation with GmMYB202 (0.41-fold) and GmMYB467 (0.84fold) (Figure 2C and Table 1).

In our co-transformation assay, six MYB TFs, GmMYB4, GmMYB77, GmMYB102, GmMYB250, GmMYB502, and GmMYB176, showed increased GUS activities for all three reporter constructs of isoflavone biosynthetic enzyme genes (i.e., GmCHS8, GmIFS1, and GmIFS2) (Figure 2), and one MYB TF, GmMYB202, suppressed GUS activity for all three reporter constructs (Figure 2). Thus, in this assay, the multiple target promoters of isoflavone biosynthetic pathway genes were differentially activated or suppressed by various MYB TFs.

These results suggest that this agroinfiltration assaywith many orphan TFs and combinations of different promoter-GUS constructs may accurately identify functional TFs for target genes on the specific and coordinately regulated biosynthetic pathway.

\section{Correlations between the expression of MYB genes and isoflavone biosynthetic enzyme genes in developing soybean seed}

To investigate correlations between total isoflavone accumulation and expression of MYB genes, isoflavone biosynthetic enzyme genes in developing soybean seed, HPLC and qRT-PCR analysis were performed. Isoflavones gradually accumulated during seed development, becoming very abundant in the late maturation stages (63 to $70 \mathrm{DAP}$ ) (Figure 3). Total isoflavone content and the expression levels of 12 candidates, GmMYB176 (positive control gene, which is known to activate GmCHS8 reporter; Yi et al. 2010), and 20 genes encoding isoflavone biosynthetic enzymes across various seed development stages, and assessed the relationships between these values by hierarchical clustering (Figure 4). The accumulation patterns of isoflavones closely correlated with the expression patterns of seven isoflavone biosynthetic enzyme genes encoding $\mathrm{CHI}$, chalcone reductase (CHR), CHS, and IFS, i.e., $G m C H I 1 A(r=0.87), G m C H R(0.95), G m C H S 1$ (0.89), GmCHS7 (0.87), GmCHS8 (0.85), GmIFS1 (0.88), and GmIFS2 (0.88); and 3 MYB genes, i.e., GmMYB102 (0.84), GmMYB280 (0.74), and GmMYB502 (0.90). The transcript levels of these genes gradually increased during seed development, with a dramatic increase at the late development stage (Figure 4; 70 DAP). The clustering results strongly suggest that the expression of GmCHS8, GmIFS1, and GmIFS2 is coordinately regulated in the soybean isoflavone biosynthetic pathway in developing seed, and that GmMYB102, GmMYB280, and GmMYB502 are putative candidates for transcriptional activators of these biosynthetic genes.
$\mathbf{A}$

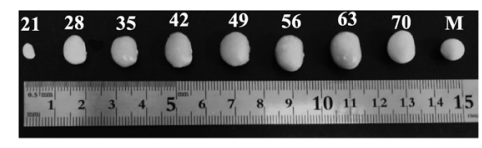

B

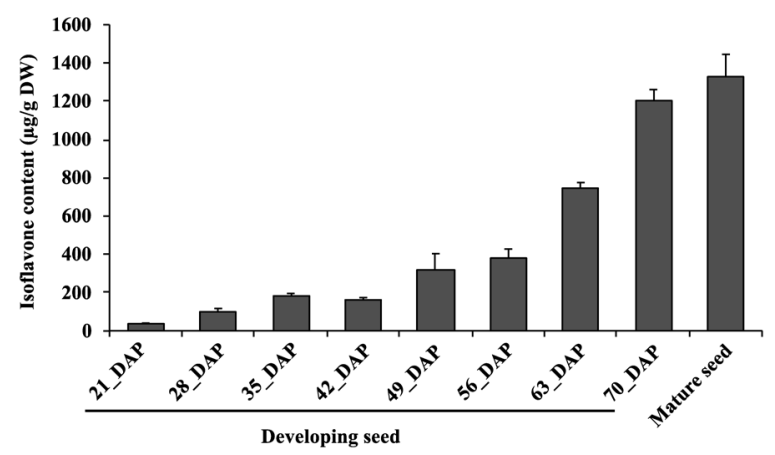

Figure 3. Morphology and total isoflavone content in developing soybean (cv. Fukuyutaka) seeds. Developing seed samples were collected at one week intervals during the period 21-70 days after pollination (DAP; A) and were used together with the mature seed sample for HPLC analysis of isoflavones (B). The data are presented as means and standard deviations of three technical replicates. DW, dry weight.

Effects of ectopic expression of MYB TFs in soybean hairy root on isoflavones and transcripts of the isoflavone biosynthetic genes

We chose three MYBs, GmMYB102, GmMYB280, and GmMYB502, for soybean hairy root transformation based on their high correlation $(r=0.84,0.74$ and 0.90 , respectively) with isoflavone accumulation in developing soybean seeds (Figure 4) and their transactivation capacity for three major isoflavone biosynthetic genes in the co-transformation assay (Figure 2). To evaluate the functional potentiality of these MYBs, we developed soybean hairy root lines, each of which overexpressed one of the above MYB genes (GmMYB102-OE, GmMYB280-OE, and GmMYB502-OE lines, respectively). By qRT-PCR analysis of the hairy root lines, we confirmed that the transcript levels of all three genes were clearly increased compared with the vector control line (Figure 5A): GmMYB102-OE ( $>1,500$-fold, $p<0.001)$, GmMYB280-OE $(>1,300$-fold, $p<0.001)$, and GmMYB502-OE ( $>30$-fold, $p<0.001$ ). We also detected an increase in total isoflavone (daidzin, glycitin, malonyldaidzin, and malonylgenistin) content in the hairy root lines GmMYB102-OE (2.6-fold, $p<0.05)$ and GmMYB502-OE $(3.8$-fold, at $p<0.01)$ compared with the control (Figure 5B); a substantial increase was also observed in the line GmMYB280-OE (2.3-fold), but this was not statistically significant. The levels of two major isoflavones (malonyldaidzin and malonylgenistin) were 2 -fold those in the control in all three hairy root lines (Figure $5 \mathrm{~B}$ ). These results suggest that at least three (GmMYB102, GmMYB280, and GmMYB502) 


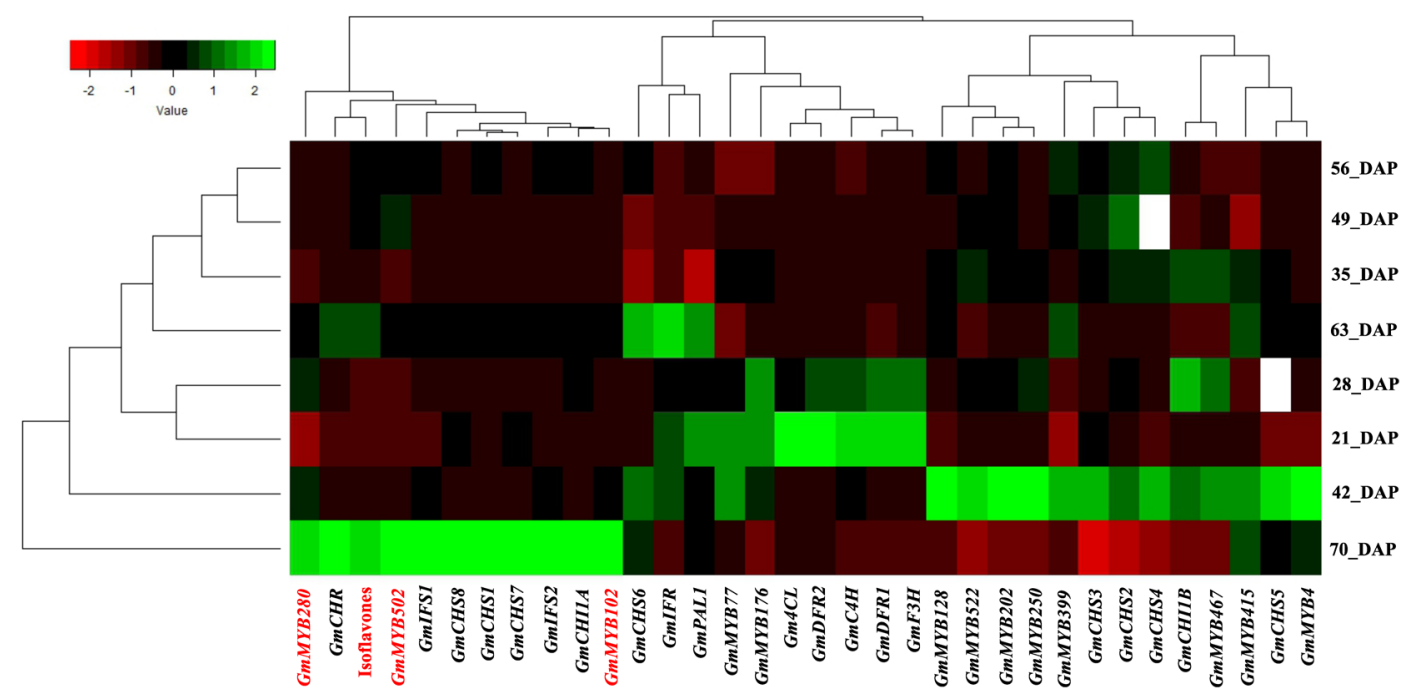

Figure 4. Hierarchical clustering and heat map analysis of gene expression profiles and the accumulation of isoflavones in developing soybean seeds. The columns represent the expression profiles of individual MYB TFs, isoflavone biosynthetic genes, and isoflavone content in developing seeds. The rows represent seed developmental stages. The white-color box in the heat map indicated that out of three replicates the expression of gene could not be detected in all the replication in qRT-PCR. The highly correlated three MYB TFs, GmMYB102, GmMYB280, GmMYB502 with isoflavones content in developing seeds are indicated by red characters. The analysis was performed in R software.

of the MYB TFs functionally identified by the soybean hairy root transformation assay could act as activators of isoflavone biosynthetic genes and increase the accumulation of target isoflavones.

\section{Discussion}

Biosynthesis and accumulation of isoflavones and related molecules require the coordinated activation or suppression of multiple genes encoding enzymes in the isoflavone biosynthetic pathway (Sablowski et al. 1994). In leguminous plants, MYB TFs transcriptionally regulate isoflavone biosynthetic genes and affect the isoflavone levels without disturbing other branches of the phenylpropanoid pathway (Yi et al. 2010; Yu et al. 2003). The R2R3-type MYB TFs consists of the largest family in plants (Dubos et al. 2010) and they play a function as transcriptional activator or repressor in diverse aspects (Dubos et al. 2010; Prouse et al. 2012). To date, R2R3MYB TFs have been identified from several plant species, such as Arabidopsis (Park et al. 2008), maize (Fornalé et al. 2010; Sonbol et al. 2009), Ginkgo (Xu et al. 2014), grape berry (Huang et al. 2014), Chrysanthemum (Zhu et al. 2013) and strawberry (Paolocci et al. 2011), which act as transcriptional regulator of several biosynthetic genes involved in the phenylpropanoid biosynthetic pathway. Here, we evaluated 12 R2R3-type MYB TFs as candidates for involvement in the isoflavone biosynthetic pathway of soybean by conducting a transient co-transformation assay and a stable hairy root transformation assay.

The co-transformation assay is known to be useful for screening both positive and candidate negative regulators from orphan TFs. However, there was a need to develop an easy and cost-effective transient multi-reporter system to reduce the false-positive detection rate.

In the transient expression assay, we analyzed the functional regulatory roles of the 12 candidate MYB TFs by using a multiple reporter system with GmCHS8, GmIFS1, and GmIFS2 promoters. CHS and IFS play a critical role in the isoflavone biosynthetic pathway of legumes and are coordinately regulated by MYB TFs (Akada and Dube 1995). Most of the MYB TFs coordinately activated or suppressed the GUS activities from multiple reporters. Out of the $12 \mathrm{MYB}$ TFs, $5 \mathrm{MYB}$ TFs, GmMYB4, GmMYB77, GmMYB102, GmMYB250, and GmMYB502, showed significant transcriptional activation of all targets tested (Figure 2).

Yi et al. (2010) reported that transient expression of $G m M Y B 176$ showed huge potential to activate the transcription of a single reporter, GmCHS8, in Arabidopsis leaf protoplasts; and RNAi silencing of GmMYB176 in soybean hairy roots resulted in reduced levels of isoflavones. In transient assays with a single reporter gene, many MYB TFs have been detected as candidates for regulators of isoflavone biosynthesis, and some of them have been confirmed to affect isoflavone accumulation in soybean (Han et al. 2017; Li et al. 2013; Yi et al. 2010). In contrast, our multiple reporter system based on the GmCHS8, GmIFS1, and GMIFS2 coordinately regulated genes in the isoflavone biosynthetic pathway, could detect MYB TFs that affected multiple metabolic reactions in this pathway; therefore, regulating these MYBs might be particularly useful for improving isoflavone accumulation.

Additionally, we used an R1-type MYB TF, GmMYB176, as a positive control to test the reliability 
A

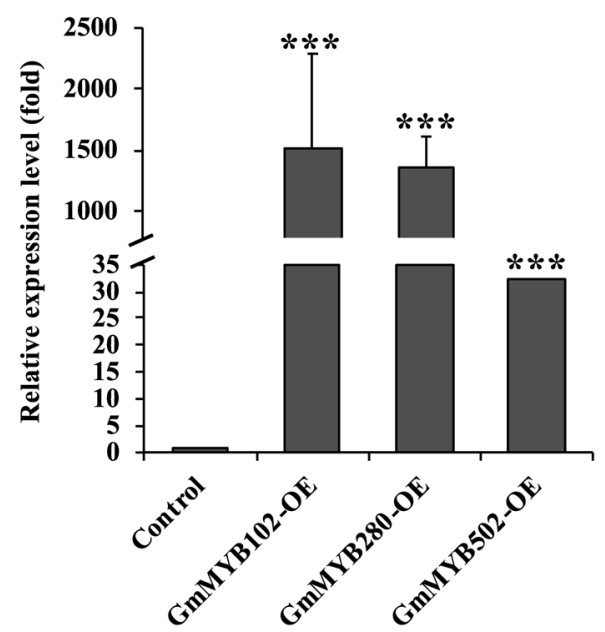

B

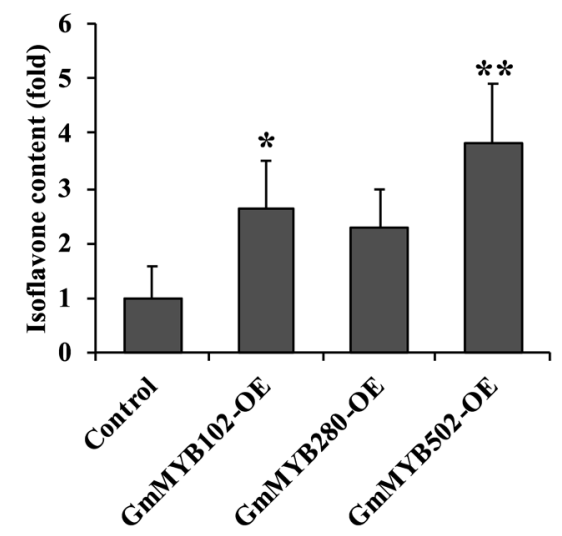

Figure 5. Isoflavone accumulation in soybean $M Y B$-overexpressing hairy root lines. Relative expression levels of $M Y B$ genes were measured in the roots of the $M Y B$-overexpressed (OS) hairy root lines and normalized with GmELFa (A). The data are presented as means \pm standard deviations of eight biological replicates. Effects of the overexpressed MYB genes on isoflavone content in the transgenic hairy roots were compared to the control (B). The data are presented as means \pm standard deviations of three independent hairy root lines. ${ }^{*} p<0.05,{ }^{* *} p<0.01$ versus an empty vector line, Student's $t$-test.

of our transient assay system. We confirmed that GmMYB176 could activate GmCHS8 reporter as previously reported (Yi et al. 2010), and we showed that it also coordinately activated both GmIFS1 and GmIFS2 reporters (Figure 2). In a recent study, combined analysis of transcripts and isoflavone contents in soybean hairy roots expressing GmMYB176-RNAi or overexpressing GmMYB176 demonstrated that GmMYB176 regulates multiple steps in isoflavone biosynthesis and affects isoflavone accumulation (Vadivel et al. 2019). These results show that our co-transformation system has great potential for evaluating the effects of orphan TFs on multiple target genes in complex metabolic pathways like isoflavone biosynthesis.

GmMYB202 significantly suppressed GUS activities of all tested target reporters. One possible explanation of this result is that there are diverse functional roles of MYB TFs and some negatively regulate transcription. Several R2R3-type MYB TFs that negatively regulate target gene transcription have been reported. GmMYB39 negatively regulated the transcription of GmCHS8 reporter in a co-transfection assay and reduced the total isoflavone level in soybean hairy roots (Liu et al. 2013). In a dual luciferase assay system, GmMYB100 suppressed the transcript levels of both GmCHS7 and $\mathrm{GmCHI}$ reporters and decreased levels of isoflavones (Yan et al. 2015). Therefore, agroinfiltration assays might be detectable both positive and negative candidate regulators from orphan TFs.

Furthermore, we demonstrated that three of these MYB genes, GmMYB102, GmMYB280, and GmMYB502 and seven isoflavone biosynthetic genes, GmCHS1, GmCHS7, GmCHS8, GmCHR, GmCHI1A, GmIFS1, and GmIFS2 were highly transcribed at the late stage (70 DAP) of seed development, i.e., the stage with the highest isoflavone accumulation (Figure 4). It has been reported that the expression patterns of the major isoflavone biosynthetic genes CHS7, CHS8, IFS1, and IFS2 correlate with the isoflavone accumulation pattern in the late stage of soybean seed development (Dhaubhadel et al. 2003, 2007). The results of the transient assay and the expression profile analysis suggested that GmMYB102, GmMYB280, and GmMYB502 are likely seed-specific activators of the isoflavone biosynthetic pathway.

MYB TFs can regulate isoflavone biosynthetic genes through binding to the target DNA binding sites of the upstream promoter region (Solano et al. 1995). We tried to find the common DNA-binding motifs in each promoter for GmCHS8, GmIFS1, or GmIFS2 by searching the PLACE database (www.dna.affrc.go.jp/PLACE/). This program predicted three type1 MYBCORE elements (CNGTTR) located in the $-388 \mathrm{bp},-453 \mathrm{bp}$, and $-1005 \mathrm{bp}$ regions of the GmCHS8 promoter (1662 bp), four MYBCORE elements located in the $-387 \mathrm{bp}$, $-1328 \mathrm{bp},-1354 \mathrm{bp}$, and $-1567 \mathrm{bp}$ regions of the GmIFS1 promoter (1740 bp), and a single MYBCORE element located in the -1245 bp region of the GmIFS2 promoter (1547bp). MYBCORE elements consist of two sub-groups (type1 and type2); in a previous report, Arabidopsis R2R3-MYBs were shown to display high binding affinity to the type1 MYBCORE elements in a yeast one-hybrid system (Kelemen et al. 2015). Therefore, type1 MYBCORE elements could be involved in the coordinate expression of GmCHS8, GmIFS1, and GmIFS2 genes via specific MYB TFs in soybean.

Finally, we confirmed activation of isoflavone biosynthesis in the roots of all the soybean hairy root lines overexpressing the three putative MYB TFs, i.e., GmMYB102-OE, GmMYB280-OE, and GmMYB502OE. In these hairy roots, ectopically expressed MYB TFs may induce the expression of multiple endogenous genes in the isoflavone biosynthetic pathway, which in turn may enhance isoflavone accumulation. We also analyzed the transcripts of several isoflavone biosynthetic 
enzyme genes in the above lines. The GmMYB502-OE hairy root line displayed substantial overexpression of genes encoding isoflavone biosynthetic enzymes, but the other two overexpressing lines showed only limited effects on the induction of these genes (Supplementary Figure S2). Recently, by conducting transcriptome analysis of GmMYB176-overexpressing hairy roots, Vadivel et al. (2019) identified several differentially expressed isoflavone biosynthetic gene families; among them, 16 were down-regulated and 6 were upregulated, suggesting dual roles for GmMYB176 as a positive and negative regulator of isoflavone biosynthetic genes. Possible explanations of some irregular gene expressions in our hairy root experiments may be that some other unidentified endogenous factor could be involved in the regulation of biosynthetic pathway gene expression and/or some other metabolic pathways of glyceollins etc. (Lygin et al. 2013) are also affected by overexpressed MYB TFs. However, we consider that the change of accumulation patterns of isoflavones in transgenic hairy roots reflects the activation or suppression of their biosynthetic pathways by MYB TFs.

Novel mutant resource development with reverse genetics and genome editing technologies instead of transgenic technology is a current trend for improving various crops, but the development of a simple and efficient method to identify the key regulator TF in a specific biosynthetic pathway as the target is still desired. In recent years, Agrobacterium-mediated transient assays have become more widely used and are considered to be an excellent substitute to stable transformation due to their easy operation and high transformation efficiency (Tsuda et al. 2012; Wroblewski et al. 2005). In comparison, the functional analysis of plant TFs by Agrobacterium-mediated stable transformation is timeconsuming and typically requires several months to obtain a dozen $\mathrm{T} 1$ transgenic plants for further analysis (Li et al. 2009; Yang et al. 2000). Our system could provide a more practical and easy way to analyze the function of orphan TFs by combining a highly efficient and reproducible transient agroinfiltration assay with a reliable hairy root transformation assay.

Therefore, we propose this system as a rapid, simple alternative to stable transformation for transcriptional activity evaluation of orphan TFs that regulate multiple target genes in a specific biosynthetic pathway in common plant species.

\section{Acknowledgements}

This research was supported in part by a Grant-in-Aid for Scientific Research (C) (Grant No. 25450009) to T. A. from the Ministry of Education, Culture, Sports, Science and Technology and Japan Society for the Promotion of Science (JSPS).

\section{References}

Akada S, Dube SK (1995) Organization of soybean chalcone synthase gene clusters and characterization of a new member of the family. Plant Mol Biol 29: 189-199

Akashi T, Aoki T, Ayabe S (1999) Cloning and functional expression of a cytochrome P450 cDNA encoding 2hydroxyisoflavanone synthase involved in biosynthesis of the isoflavonoid skeleton in licorice. Plant Physiol 121: 821-828

Aoki T, Akashi T, Ayabe S (2000) Flavonoids of leguminous plants: Structure, biological activity, and biosynthesis. J Plant Res 113: 475-488

Aoyagi LN, Lopes-Caitar VS, de Carvalho MCCG, Darben LM, Polizel-Podanosqui A, Kuwahara MK, Nepomuceno AL, Abdelnoor RV, Marcelino-Guimarães FC (2014) Genomic and transcriptomic characterization of the transcription factor family R2R3-MYB in soybean and its involvement in the resistance responses to Phakopsora pachyrhizi. Plant Sci 229: 32-42

Berger B, Stracke R, Yatusevich R, Weisshaar B, Flügge UI, Gigolashvili T (2007) A simplified method for the analysis of transcription factor-promoter interactions that allows highthroughput data generation. Plant J 50: 911-916

Bradford MM (1976) A rapid and sensitive for the quantitation of microgram quantities of protein utilizing the principle of protein-dye binding. Anal Biochem 72: 248-254

Broun P (2004) Transcription factors as tools for metabolic engineering in plants. Curr Opin Plant Biol 7: 202-209

Chen L, Cai Y, Liu X, Guo C, Sun S, Wu C, Jiang B, Han T, Hou W (2018) Soybean hairy roots produced in vitro by Agrobacterium rhizogenes-mediated transformation. Crop J 6: 162-171

Dastmalchi M, Dhaubhadel S (2015) Soybean chalcone isomerase: Evolution of the fold, and the differential expression and localization of the gene family. Planta 241: 507-523

Dhaubhadel S, Gijzen M, Moy P, Farhangkhoee M (2007) Transcriptome analysis reveals a critical role of CHS7 and CHS8 genes for isoflavonoid synthesis in soybean seeds. Plant Physiol 143: 326-338

Dhaubhadel S, McGarvey BD, Williams R, Gijzen M (2003) Isoflavonoid biosynthesis and accumulation in developing soybean seeds. Plant Mol Biol 53: 733-743

Dixon RA (2004) Phytoestrogen. Annu Rev Plant Biol 55: 225-261

Du YT, Zhao MJ, Wang CT, Gao Y, Wang YX, Liu YW, Chen M, Chen J, Zhou YB, Xu ZS, et al. (2018) Identification and characterization of GmMYB118 responses to drought and salt stress. BMC Plant Biol 18: 320

Dubos C, Stracke R, Grotewold E, Weisshaar B, Martin C, Lepiniec L (2010) MYB transcription factors in Arabidopsis. Trends Plant Sci 15: 573-581

Ferguson BJ, Mathesius U (2003) Signaling interactions during nodule development. J Plant Growth Regul 22: 47-72

Fornalé S, Shi X, Chai C, Encina A, Irar S, Capellades M, Fuguet E, Torres JL, Rovira P, Puigdomènech P, et al. (2010) ZmMYB31 directly represses maize lignin genes and redirects the phenylpropanoid metabolic flux. Plant J 64: 633-644

Grotewold E, Sainz MB, Tagliani L, Hernandez JM, Bowen B, Chandler VL (2000) Identification of the residues in the Myb domain of maize $\mathrm{C} 1$ that specify the interaction with the bHLH cofactor R. Proc Natl Acad Sci USA 97: 13579-13584

Han X, Yin Q, Liu J, Jiang W, Di S, Pang Y (2017) GmMYB58 and GmMYB205 are seed-specific activators for isoflavonoid biosynthesis in Glycine max. Plant Cell Rep 36: 1889-1902

Holsters M, de Waele D, Depicker A, Messens E, van Montagu 
M, Schell J (1978) Transfection and transformation of Agrobacterium tumefaciens. Mol Gen Genet 163: 181-187

Huang YF, Vialet S, Guiraud JL, Torregrosa L, Bertrand Y, Cheynier V, This P, Terrier N (2014) A negative MYB regulator of proanthocyanidin accumulation, identified through expression quantitative locus mapping in the grape berry. New Phytol 201: 795-809

Jefferson RA (1987) Assaying chimeric genes in plants: The GUS gene fusion system. Plant Mol Biol Report 5: 387-405

Jefferson RA, Burgess SM, Hirsh D (1986) Beta-Glucuronidase from Escherichia coli as a gene-fusion marker. Proc Natl Acad Sci USA 83: 8447-8451

Jung W, Yu O, Lau SMC, O'keefe DP, Odell J, Fader G, McGonigle B (2000) Identification and expression of isoflavone synthase, the key enzyme for biosynthesis of isoflavones in legumes. Nat Biotechnol 18: 208-212

Kapila J, Rycke RD, van Montagu M, Angenon G (1997) An Agrobacterium-mediated transient gene expression system for intact leaves. Plant Sci 122: 101-108

Kelemen Z, Sebastian A, Xu W, Grain D, Salsac F, Avon A, Berger N, Tran J, Dubreucq B, Lurin C, et al. (2015) Analysis of the DNA-binding activities of the Arabidopsis R2R3-MYB transcription factor family by one-hybrid experiments in yeast. PLoS One 10: e0141044

Kereszt A, Li D, Indrasumunar A, Nguyen CD, Nontachaiyapoom S, Kinkema M, Gresshoff PM (2007) Agrobacterium rhizogenesmediated transformation of soybean to study root biology. Nat Protoc 2: 948-952

Kosslak RM, Bookland R, Barkei J, Paaren HE, Appelbaum ER (1987) Induction of Bradyrhizobium japonicum common nod genes by isoflavones isolated from Glycine max. Proc Natl Acad Sci USA 84: 7428-7432

Lam H-M, Xu X, Liu X, Chen W, Yang G, Wong F-L, Li M-W, He W, Qin N, Wang B, et al. (2010) Resequencing of 31 wild and cultivated soybean genomes identifies patterns of genetic diversity and selection. Nat Genet 42: 1053-1059

Latunde-Dada AO, Cabello-Hurtado F, Czittrich N, Didierjean L, Schopfer C, Hertkorn N, Werck-Reichhart D, Ebel J (2001) Flavonoid 6-hydroxylase from soybean (Glycine max L.), a novel plant P-450 monooxygenase. J Biol Chem 276: 1688-1695

Li JF, Park E, von Arnim AG, Nebenführ A (2009) The FAST technique: A simplified Agrobacterium-based transformation method for transient gene expression analysis in seedlings of Arabidopsis and other plant species. Plant Methods 5: 6

Li XW, Li JW, Zhai Y, Zhao Y, Zhao X, Zhang HJ, Su LT, Wang Y, Wang QY (2013) A R2R3-MYB transcription factor, $G m M Y B 12 B 2$, affects the expression levels of flavonoid biosynthesis genes encoding key enzymes in transgenic Arabidopsis plants. Gene 532: 72-79

Liu X, Yuan L, Xu L, Xu Z, Huang Y, He X, Ma H, Yi J, Zhang D (2013) Over-expression of GmMYB39 leads to an inhibition of the isoflavonoid biosynthesis in soybean (Glycine max. L). Plant Biotechnol Rep 7: 445-455

Livak KJ, Schmittegen TD (2001) Analysis of relative gene expression data using real-time quantitative PCR and the $2^{-\Delta \Delta \mathrm{C}_{\mathrm{T}}}$ ) method. Methods 25: 402-408

Lygin AV, Zernova OV, Hill CB, Kholina NA, Widholm JM, Hartman GL, Lozovaya VV (2013) Glyceollin is an important component of soybean plant defense against Phytophthora sojae and Macrophomina phaseolina. Phytopathology 103: 984-994

Maekawa T, Kusakabe M, Shimoda Y, Sato S, Tabata S, Murooka Y, Hayashi M (2008) Polyubiquitin promoter-based binary vectors for overexpression and gene silencing in Lotus japonicus. Mol Plant Microbe Interact 21: 375-382

Messina MJ (1999) Legumes and soybeans: Overview of their nutritional profiles and health effects. Am J Clin Nutr 70(Suppl): 439S-450S

Nesi N, Jond C, Debeaujon I, Caboche M, Lepiniec L (2001) The Arabidopsis TT2 gene encodes an R2R3 MYB domain protein that acts as a key determinant for proanthocyanidin accumulation in developing seed. Plant Cell 13: 2099-2114

Nestel PJ, Pomeroy S, Kay S, Komesaroff P, Behrsing J, Cameron JD, West L (1999) Isoflavones from red clover improve systemic arterial compliance but not plasma lipids in menopausal women. J Clin Endocrinol Metab 84: 895-898

Newman LJ, Perazza DE, Juda L, Campbell MM (2004) Involvement of the R2R3-MYB, AtMYB61, in the ectopic lignification and dark-photomorphogenic components of the det3 mutant phenotype. Plant J 37: 239-250

Novák K, Lisá L, Škrdleta V (2004) Rhizobial nod gene-inducing activity in pea nodulation mutants: Dissociation of nodulation and flavonoid response. Physiol Plant 120: 546-555

Paolocci F, Robbins MP, Passeri V, Hauck B, Morris P, Rubini A, Arcioni S, Damiani F (2011) The strawberry transcription factor FaMYB1 inhibits the biosynthesis of proanthocyanidins in Lotus corniculatus leaves. J Exp Bot 62: 1189-1200

Park JS, Kim JB, Cho KJ, Cheon CI, Sung MK, Choung MG, Roh KH (2008) Arabidopsis R2R3-MYB transcription factor AtMYB60 functions as a transcriptional repressor of anthocyanin biosynthesis in lettuce (Lactuca sativa). Plant Cell Rep 27: 985-994

Peterson G, Barnes S (1991) Genistein inhibition of the growth of human breast cancer cells: Independence from estrogen receptors and the multi-drug resistance gene. Biochem Biophys Res Commun 179: 661-667

Potter SM, Baum JA, Teng H, Stillman RJ, Shay NF, Erdman JW Jr (1998) Soy protein and isoflavones: Their effects on blood lipids and bone density in postmenopausal women. Am J Clin Nutr 68(Suppl): 1375S-1379S

Prouse MB, Campbell MM (2012) The interaction between MYB proteins and their target DNA binding sites. Biochim Biophys Acta 1819: 67-77

Ramsay NA, Glover BJ (2005) MYB-bHLH-WD40 protein complex and the evolution of cellular diversity. Trends Plant Sci 10: 63-70

Ron M, Kajala K, Pauluzzi G, Wang D, Reynoso MA, Zumstein K, Garcha J, Winte S, Masson H, Inagaki S, et al. (2014) Hairy root transformation using Agrobacterium rhizogenes as a tool for exploring cell type-specific gene expression and function using tomato as a model. Plant Physiol 166: 455-469

Sablowski RW, Moyano E, Culianez-Macia FA, Schuch W, Martin C, Bevan M (1994) A flower-specific Myb protein activates transcription of phenylpropanoid biosynthetic genes. EMBO J 13: $128-137$

Schmutz J, Chapman J, Hellsten U, Rokhsar D (2008) Sequence and Assembly of the Soybean Genome. In: Stacey G (ed) Genetics and Genomics of Soybean. Plant Genetics and Genomics: Crops and Models, vol 2. Springer, New York, NY

Solano R, Nieto C, Avila J, Cañas L, Diaz I, Paz-Ares J (1995) Dual DNA binding specificity of a petal epidermis-specific MYB transcription factor (MYB.Ph3) from Petunia hybrida. EMBO J 14: 1773-1784

Sonbol FM, Fornalé S, Capellades M, Encina A, Touriño S, Torres JL, Rovira P, Ruel K, Puigdomènech P, Rigau J, et al. (2009) The maize ZmMYB42 represses the phenylpropanoid pathway and 
affects the cell wall structure, composition and degradability in Arabidopsis thaliana. Plant Mol Biol 70: 283-296

Steele CL, Gijzen M, Qutob D, Dixon RA (1999) Molecular characterization of the enzyme catalyzing the aryl migration reaction of isoflavonoid biosynthesis in soybean. Arch Biochem Biophys 367: 146-150

Tsuda K, Qi Y, Nguyen LV, Bethke G, Tsuda Y, Glazebrook J, Katagiri F (2012) An efficient Agrobacterium-mediated transient transformation of Arabidopsis. Plant J 69: 713-719

Vadivel AKA, Renaud J, Kagale S, Dhaubhadel S (2019) GmMYB176 regulates multiple steps in isoflavonoid biosynthesis in soybean. Front Plant Sci 10: 562

Wroblewski T, Tomczak A, Michelmore R (2005) Optimization of Agrobacterium-mediated transient assays of gene expression in lettuce, tomato and Arabidopsis. Plant Biotechnol J 3: 259-273

Xu F, Ning Y, Zhang W, Liao Y, Li L, Cheng H, Cheng S (2014) An R2R3-MYB transcription factor as a negative regulator of the flavonoid biosynthesis pathway in Ginkgo biloba. Funct Integr Genomics 14: 177-189

Yan J, Wang B, Zhong Y, Yao L, Cheng L, Wu T (2015) The soybean R2R3 MYB transcription factor $G m M Y B 100$ negatively regulates plant flavonoid biosynthesis. Plant Mol Biol 89: 35-48

Yang Y, Li R, Qi M (2000) In vivo analysis of plant promoters and transcription factors by agroinfiltration of tobacco leaves. Plant J 22: $543-551$
Ye J, Duan Y, Hu G, Geng X, Zhang G, Yan P, Liu Z, Zhang L, Song $X$ (2017) Identification of candidate genes and biosynthesis pathways related to fertility conversion by wheat KTM3315A transcriptome profiling. Front Plant Sci 8: 449

Yi J, Derynck MR, Li X, Telmer P, Marsolais F, Dhaubhadel S (2010) A single-repeat MYB transcription factor, GmMYB176, regulates CHS8 gene expression and affects isoflavonoid biosynthesis in soybean. Plant J 62: 1019-1034

Yoneyama K, Akashi T, Aoki T (2016) Molecular characterization of soybean pterocarpan 2-dimethylallyltransferase in glyceollin biosynthesis: Local gene and whole-genome duplications of prenyltransferase genes led to the structural diversity of soybean prenylated isoflavonoids. Plant Cell Physiol 57: 2497-2509

Yu O, Shi J, Hession AO, Maxwell AA, McGonigle B, Odell JT (2003) Metabolic engineering to increase isoflavone biosynthesis in soybean seeds. Phytochemistry 63: 753-763

Zhou J, Lee C, Zhong R, Ye ZH (2009) MYB58 and MYB63 are transcriptional activators of the lignin biosynthetic pathway during secondary cell wall formation in Arabidopsis. Plant Cell 21: $248-266$

Zhu L, Shan H, Chen S, Jiang J, Gu C, Zhou G, Chen Y, Song A, Chen F (2013) The Heterologous Expression of the Chrysanthemum R2R3-MYB Transcription Factor CmMYB1 Alters Lignin Composition and Represses Flavonoid Synthesis in Arabidopsis thaliana. PLoS One 8: e65680 